\title{
Anabases
}

ANABASES Traditions et réceptions de l'Antiquité

6 | 2007

Varia

\section{La corruption, un concept philosophique et politique chez les Anciens et les Modernes.} Introduction

Thierry Ménissier

\section{(2) OpenEdition}

\section{Journals}

Édition électronique

URL : http://journals.openedition.org/anabases/3284

DOI : $10.4000 /$ anabases.3284

ISSN : 2256-9421

Éditeur

E.R.A.S.M.E.

Édition imprimée

Date de publication : 1 octobre 2007

Pagination : 11-16

ISSN : 1774-4296

\section{Référence électronique}

Thierry Ménissier, "La corruption, un concept philosophique et politique chez les Anciens et les Modernes. », Anabases [En ligne], 6 | 2007, mis en ligne le 01 janvier 2012, consulté le 20 octobre 2019. URL : http://journals.openedition.org/anabases/3284; DOI : 10.4000/anabases.3284

(c) Anabases 
Anabases 6 (2007), p. 11-16.

\section{La corruption, un concept philosophique et politique chez les Anciens et les Modernes. Introduction *}

THIERRY MÉNISSIER

C' EST UNE BIEN REDOUTABLE NOTION que celle de la corruption. Aussi intuitivement parlante que mal définie, on peine à décider si elle consiste en une métaphore ou si elle est susceptible d'être utilisée comme un concept, une catégorie claire et distincte capable d'appréhender rationnellement la réalité. Défiant les cloisonnements académiques, elle évoque des réalités multiples et variées pour l'historien comme pour le sociologue, pour le juriste comme pour le philosophe, sinon pour le psychologue et pour l'anthropologue. De plus, elle divise et rapproche tour à tour les Anciens et les Modernes, selon qu'on l'envisage en tant que notion philosophique ou sur le terrain de la théorie sociale et politique.

Considérée sur le plan des idées philosophiques, d'abord, la notion de corruption semble diviser les Anciens et les Modernes. Les " physiciens » de la Grèce l'ont envisagée sous l'angle de la nature des choses, en tant qu'altération progressive et inéluctable des êtres naturels ( $p h t o r a)$. Reflet et synthèse de cette tradition, le traité aristotélicien De la génération et de la corruption estime que cette dernière constitue, avec la génésis, une des qualités fondamentales des êtres qui évoluent dans le monde sublunaire. En permettant l'assignation de ces êtres à une certaine idée de la nature (phusis), la notion de corruption participe à un repérage ontologique : c'est-à-dire qu'elle qualifie un certain type d'être dans sa nature essentielle, et que cette nature, soumise au temps et à l'altération, est perçue comme mouvante et dynamique ${ }^{1}$. Ensuite s'ouvre un registre

* Ce dossier d'articles est issu de la journée d'étude qui s'est déroulée à l'Université de Toulouse-Le Mirail le 19 janvier 2007. Je remercie particulièrement Pascal Payen et Marie-Laurence Desclos de m'avoir permis de l'organiser.

1

Voir Aristote, De la génération et de la corruption, particulièrement I, 3, 317 a 31-319 b 5, et II, 9-10, 335 a 23-337 a 33. 
très différent d'une telle saisie conceptuelle, un registre d'origine religieuse et tragique, mais lié à la physique des Anciens par le biais de certaines pratiques purgatives. La corruption évoque le thème de l'impureté, ce qui permet d'interroger le complexe travail des métaphores connexes. Dans une importante note critique, Jean-Pierre Vernant mettait en relief la manière dont elles jouent dans le texte tragique, de l'évocation de la saleté à celle de la souillure ${ }^{2}$. Il existe encore une troisième modalité d'expression de la notion de corruption dans l'Antiquité, qui se trouve associée aux deux précédentes sans pouvoir s'y réduire : celle qui procède par rapprochement avec le thème de la maladie. La riche métaphore pathologique, il est permis de l'appréhender par le biais de sa fonction; elle sert manifestement à qualifier certaines tendances ou certains états humains, et trouve son emploi dans le champ de l'axiologie, qu'il s'agisse, dans les domaines moral, social et politique, d'évoquer l'indignité ou l'insalubrité de certaines pratiques, ou bien de souligner, par la désignation a contrario de certaines valeurs positives, les limites que la condition humaine ne saurait dépasser sans se dénaturer ${ }^{3}$.

Ce rapide repérage donne à penser que, dans les trois cas, les Anciens paraissent avoir développé une représentation de la corruption qu'à défaut de qualifier de "positive " on pourrait nommer " efficace ", en ce que, descriptive et normative, elle permet à l'homme d'évaluer sa conduite individuelle et collective, mais aussi d'agir sur elle. Très différente est au départ la notion moderne, qui se saisit initialement dans l'anthropologie chrétienne construite autour de l'épisode du péché originel. Tout aussi prescriptive, la notion de corruption se voit alors employée afin de qualifier, sur tous les plans de l'existence humaine, les efforts d'un être humain voué à un destin paradoxal 4 . Stigmatisé dès le début de son existence en tant qu'espèce, l'homme est appelé à déployer toutes ses ressources possibles afin d'exprimer dans son existence singulière comme dans l'histoire tout entière ce qui le fait ressembler à Dieu, à l'image duquel il

2 Jean-Pierre VernANT, "Le pur et l'impur" (qui fait la recension de l'ouvrage de L. Moulinier, Le pur et l'impur dans la pensée et la sensibilité des Grecs jusquà la fin du IVe siècle avant J.-C., Paris, Klincksieck, 1952), paru dans L'année sociologique, 1953-54, repris dans Mythe et société en Grèce ancienne, Paris, La Découverte, 1974 ; rééd. Le Seuil, "Points essais ", p. 121-140.

3 À propos de la manière dont Thucydide et Platon exploitent chacun à leur manière le répertoire des thèmes associés, voir respectivement Marie-Laurence DesClos, Aux marges des dialogues de Platon. Essai d'histoire anthropologique de la philosophie ancienne, Grenoble, Jérôme Millon, 2003, particulièrement le chapitre V: "Thucydide et la maladie de la cité", p. 87-120 ; et Thierry MÉNISSIER, "Platon et le concept de maladie dans la République", Études philosophiques, n 3 de 1995, p. 355-373. telle anthropologie, cf. la thèse de François BUSSINI, L'homme pécheur devant Dieu : théologie et anthropologie, Thèse de doctorat de théologie catholique, Lille, Service de Reproduction des Thèses de l'Université, 1979. 
fut créé 5 . Malgré les apparences, le thème de la corruption ainsi décliné sépare profondément les Modernes des Anciens, pour lesquels il serait contradictoire de considérer l'humanité ainsi stigmatisée par nature. Mais un tel cadre n'a jamais été statique, ni la doctrine, absolument figée : à l'inverse, dans toutes les époques de la pensée chrétienne, de constantes réélaborations du schéma initial se sont jouées par le biais de la spéculation théologique. On en trouvera dans ce dossier deux exemples privilégiés. D’abord, dans son étude " 'Ce qui fut en lui était la vie' (Jn 1, 3-4) : que signifie une vie qui ne se corrompt pas ? Réflexion sur la vie dans le principe chez Albert le Grand et Michel Henry", Julie Casteigt montre que le prologue de l'Évangile selon saint Jean comprend une idée de vie incorruptible susceptible de faire dialoguer, par-delà les siècles, le commentaire d'Albert le Grand et l'approche phénoménologique de Michel Henry. Ensuite, parce qu'elle est à la fois issue d'un contexte théologique qui trouve son origine lointaine dans la pensée augustinienne et construite autour de la méditation des auteurs de l'Antiquité, l'œuvre philosophique d'un Pascal, telle qu'Anne Frostin-Garreta l'envisage ci-dessous dans son étude "Vie et belle santé de la corruption dans la politique de Pascal ", représente un cas de figure exemplaire de la tension entre deux conceptions de l'homme et de la corruption.

En revanche, dans l'histoire des idées politiques, la notion de corruption parât certes avoir servi des objectifs variés, mais capables d'associer étroitement les Anciens et les Modernes. D'une part, telle que les penseurs politiques anciens et modernes (de Platon à Montesquieu) l'ont entendue, elle est perversion ou pathologie des régimes. Cet usage de la notion paraît être la conséquence d'une représentation naturaliste de la logique politique, et se trouve employée dans le cadre des métaphores vitalistes ou médicales évoquées plus haut. La transformation d'un régime en un autre est conçue comme altération d'un corps physique ou d'un organisme vivant. Une telle représentation semble de plus avoir permis de concevoir la temporalité spécifique de la vie politique, sinon la nature du devenir historique, ainsi qu'on le découvre chez des auteurs dont les intentions sont variées, tels Thucydide, Platon, Aristote, Polybe, voire Machiavel ${ }^{6}$. D'autre part, fort différemment, la notion de corruption a valu d'un point de vue éthique, dans le but d'appréhender la rectitude des conduites socio-politiques individuelles et collectives : selon cet usage, sont désignées comme corrompues certaines formes d'inconduite. Il faudrait déterminer lesquelles et pour quelles raisons - ce qui revient à examiner comment un discours théorique est à même de juger des situations sociales variées, telles que le clientélisme et l'évergétisme pour les Anciens, et la vénalité des offices pour les Modernes. Or, non seulement ces pratiques constituent souvent de véritables systèmes d'organisation, mais encore une approche sociologique de type

5 Le double plan du salut est particulièrement signalé par Saint Augustin dans La cité de Dieu, voir notamment X, 14 et XIII, 12-15

6 Voir la synthèse de Norberto BoBBIO, La teoria delle forme di governo nella storia del pensiero politico, Cours universitaire de l'année 1975-76, Turin, Giappichelli, 1976. 
fonctionnaliste leur reconnaît toujours une raison d'être, voire une pertinence spécifique ${ }^{7}$. Dans ce cas de figure, toute entreprise théorique d'imputation de corruption parait devoir préalablement adopter une représentation normative du comportement civique, nécessaire pour que l'on puisse parler d'inconduite. C'est la raison pour laquelle l'analyse de cet usage de la notion de corruption nous conduit aux racines mêmes de la caractérisation éthique.

De nombreux philosophes anciens et modernes ont procédé de la sorte, mais peut-être pas tous dans le même dessein : ainsi Platon dans les livres VIII et IX de $L a$ République, Aristote dans ses réflexions sur la tyrannie du livre V de la Politique, Montesquieu avec son combat contre le despotisme en restituant dans De l'esprit des lois la logique qui conduit le «vizir » à capter à son profit le pouvoir public. La plupart de ces auteurs raisonnent sinon en personnifiant la corruption, du moins en constituant une figure du tyran ou du despote qui leur permet de dessiner en contrepoint les conduites privées et publiques "saines " ou régulières. Une telle manière de réfléchir revient de surcroît à assurer l'analyse du geste politique par une détermination morale : l'évaluation de la conduite politiquement corrompue s'effectue plus ou moins clairement en fonction d'une représentation de la vertu et du vice. Le thème de la corruption ainsi envisagé semble même se situer à l'intersection de deux problématiques hétérogènes : il fournit un schème d'intelligibilité des pratiques éthiques et politiques en combinant la métaphore pathologique - qui naturalise les comportements d'inconduite - et la thématique typiquement moderne du péché - qui repose sur une anthropologie fortement déterminée par des valeurs utilisées de manière absolue. Ainsi est-il opportun de démêler ce que l'idée de corruption doit à la théologie, ou comment une thématique initialement médicale a pu, dans la modernité, accueillir certains éléments d'origine théologique. Comment le thème de la dépravation des mœurs, au confluent des problématiques médicale et théologique, a-t-il évolué dans la pensée historique et philosophique moderne, par exemple dans des contextes de rupture comme celui de la Révolution française ? Joue-t-il un rôle décisif dans la reformulation de la morale privée et publique des Modernes? On verra comment Thomas Van der Hallen aborde ces questions à partir de l'examen du cas privilégié que représente le discours de celui qu'on nommait "l'Incorruptible ", dans son étude "Corruption et régénération du politique chez Robespierre".

Ces manières d'envisager la corruption furent élaborées au sein d'entreprises théoriques, mais on peut également déceler dans l'histoire des représentations esthétiques un travail d'élaboration intellectuel très intéressant. Ainsi que l'établit Bertrand Prévost dans son étude "Rome-Babylone. Ruine, corruption, colosse", le thème pictural de la

7 Voir Robert K. MERTON, Éléments de théorie et de méthode sociologique [1957], trad. Henri Mendras, Paris, Plon, 2e édition 1965, particulièrement le chapitre III, "Lanalyse fonctionnelle en sociologie", p. 60-135. 
ruine, singulièrement, paraît avoir fait fonction de schème de formulation d'une idée de corruption dont il est nécessaire de formuler la nature et d'envisager la portée.

Il est également nécessaire, bien entendu, de se demander comment, chez les Anciens et les Modernes, se définit l'idée proprement juridico-politique de corruption. Pour les premiers, la démocratie athénienne fournit un cas d'espèce à l'étude, notamment du fait de son exécrable réputation, œuvre des poètes comiques aussi bien que des philosophes ; l'examen des dispositifs constitutionnels athéniens offre pourtant un moyen privilégié si l'on veut déterminer une idée sinon une norme de corruption saisie à l'intersection des règles juridico-politiques, des pratiques sociales et des représentations collectives ${ }^{8}$. En ce sens, les Athéniens paraissent avoir inventé cette forme d'esprit sans lequel la démocratie n'est qu'un mot, quand bien même cette invention fut complexe, et à certains égards paradoxale car effectuée sous l'effet de dispositifs contraints, ainsi qu'on le voit avec le développement de la délation par le biais des sycophantes, ou accusation publique volontaire ${ }^{9}$. Chez les Modernes, une semblable norme paraît explicitement avoir été engendrée par le républicanisme moderne, luimême lointain héritier du discours républicain ancien revivifié par les pratiques « révolutionnaires " des expériences florentine, américaine et française. On décèle chez Machiavel les prémisses de cette manière de penser, qui s'est affinée et diversifiée dans les discours sur la vertu civique jusqu'aux œuvres de Montesquieu et de Rousseau : par le biais de récits de l'histoire de Rome et de Florence (dans les Discours sur la première décade de Tite-Live et dans les Histoires florentines), et grâce à la confrontation systématique du paradigme ancien et de son prétendu correspondant moderne, Machiavel a établi le profil de l'inconduite civique en narrant l'entrelacement des motifs privés et des actions publiques. La détermination de la notion de corruption s'effectue à même l'appréhension des conduites dans l'espace des cités-États, et toujours en fonction de la capacité de celles-ci à trouver des formes d'organisation susceptibles de les voir échapper aux tumultes internes et aux invasions étrangères. De ce fait, Machiavel fut sans doute le premier dans la modernité à avoir envisagé un concept proprement politique de corruption: ni métaphorique, ni théologique, ni même morale, la recherche machiavélienne s'effectuait tantôt sur le plan social (lorsqu'il s'agit de repérer puis d'évaluer quelle " humeur " est néfaste à la cité), tantôt sur le plan constitutionnel et civique (lorsqu'il s'agit de sonder tel ou tel " ordre " (ordine) d'une part, et en entreprenant d'examiner les faits et gestes des acteurs de la vie publique, de l'autre). Peut-on dire que le Florentin a fait basculer l'usage de la notion dans sa période moderne ? Comment ce type de discours se poursuit-il jusqu’à la Révolution française ? Existe-t-il un lien

8 Voir Mogens Herman HANSEN, La démocratie grecque à l'époque de Démosthène. Structure, principes et idéologie, trad. S. Bardet avec le concours de P. Gauthier, Paris, Les Belles Lettres, 1993.

9 Sur les sycophantes à Athènes et sur un tel usage de la délation, voir Carine Doganis, Aux Origines de la corruption. Démocratie et délation en Grèce ancienne, Paris, PUF, 2007. 
évident entre cette manière d'appréhender la corruption et la définition purement déontologique de ce terme, telle que le Code civil de 1804 l'a présentée ? Pour avoir une idée plus précise du type de réponse qu'on peut apporter à ces questions, il semble nécessaire d'évoquer une autre dimension. En effet, un aspect fondamental de ce problème tient vraisemblablement dans la manière dont la modernité a modifié les conditions mêmes de la représentation de l'avoir. Une des dimensions de la réflexion sur la corruption consiste ici à évaluer l'écart entre les Anciens et les Modernes à propos des relations que peuvent légitimement entretenir la propriété privée, l'échange marchand et symbolique, et la constitution d'une communauté socio-politique ${ }^{10}$. Il convient de se demander si l'apparition d'un discours typiquement moderne sur le commerce - on serait tenté d'écrire : "d'un discours $d u$ commerce " - a ou non radicalement modifié les choses. La naissance du libéralisme, quelque part entre le XVII (dans la philosophie d'inspiration puritaine d'un Locke, par exemple) et le XVIII siècle (dans la reconnaissance des bienfaits du commerce par Montesquieu, notamment), n'a-t-elle pas profondément bouleversé l'ancienne conception de la corruption, au point de permettre l'apparition de son concept purement déontologique ${ }^{11}$ ? Ce sont ces questions que rencontre l'étude de Thierry Ménissier, "L'usage civique de la notion de corruption selon le républicanisme ancien et moderne ".

En fonction des éléments que nous venons de développer, les études réunies dans ce dossier s'assignent deux objectifs principaux : d'une part, celui de clarifier les conditions de l'usage de la notion de corruption chez les Anciens et les Modernes (et d'apercevoir de ce fait les lignes de continuité et les thèmes de rupture entre eux), et de l'autre d'envisager les conséquences de cet usage sur la représentation de la vie sociale et politique.

\begin{tabular}{l} 
Thierry MÉNISSIER \\
\hline UFR SH Université Pierre Mendès France-Grenoble 2 \\
Bât. Sciences de l'Homme \\
BP $47-1251$, av. Centrale \\
38040 Grenoble Cedex 9 \\
téléphone : 33476825646 \\
courriel : Thierry.Menissier@upmf-grenoble.fr
\end{tabular}

10 Sur ces thèmes, voir Vincent AzOUlay, Xénophon et les grâces du pouvoir. De la charis au charisme, Paris, Publications de la Sorbonne, 2004, qui propose une analyse des relations entre ces thèmes tels que Xénophon les pense, à partir d'une matrice de pensée héritée de l'Essai sur le don de Marcel MaUSs. Dans une perspective plus vaste, cf. l'ouvrage collectif coordonné par Dieter Haller et Cris SHORE (dir.), Corruption : Anthropological Perspectives, Pluto Press, 2005.

11 Sur l'apparition d'un tel discours, cf. Albert O. HirsChMANN, Les passions et les intérêts. Justifications politiques du capitalisme avant son apogée [1977], trad. Paris, PUF, 1980, "Quadrige ", 1997. Sur ses enjeux plus généraux, cf. Marcel Henaff, Le prix de la vérité. Le don, l'argent, la philosophie, Paris, Le Seuil, 2002. 